Prix Nobel de Chimie 2015

Thomas Lindahl, Paul Modrich et Aziz Sankar

\section{Un Nobel pour la réparation de I'ADN}

\author{
Bertrand Jordan
}

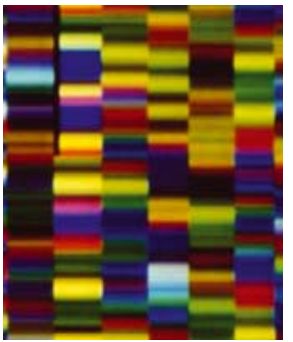

UMR 7268 ADÉS, Aix-Marseille, Université/EFS/CNRS, Espace éthique méditerranéen, hôpital d'adultes la Timone, 264, rue Saint-Pierre, 13385 Marseille Cedex 05, France ;

CoReBio PACA, case 901, parc scientifique de Luminy, 13288 Marseille Cedex 09, France. bertrand.jordan@univ-amu.fr

dénaturer ${ }^{1}$, même en l'absence de brjordan@orange.fr son intégrité. Les trois chercheurs distingués par ce prix Nobel, Thomas Lindahl, Paul Modrich et Aziz Sankar, sont arrivés à ce thème par des itinéraires très différents et ont chacun apporté des contributions fondamentales. La réparation de I'ADN constitue aujourd'hui un domaine de recherche en soi, et il n'est pas question ici d'en faire une présentation, même résumée : cela demanderait tout un volume. Je me limiterai donc à quelques indications sur chacun des lauréats et sur leur thème principal. >

\section{Trois profils bien différents}

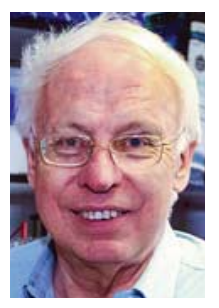

Thomas Lindahl

Né à Stockholm en 1938, Thomas Lindahl se destinait au départ à la médecine mais, après l'obtention de son diplôme, commença à s'intéresser à la biochimie des acides nucléiques sous l'influence d'Einar Hammarsten, pionnier du domaine et professeur à I'Institut Karolinska (Stockholm, Suède). II partit en 1965 faire un post-doc aux États-Unis dans le laboratoire de Jacques Fresco à Princeton, spécialisé dans l'étude des ARN de transfert (ARNt), et s'y heurta à l'instabilité intrinsèque de ces molécules lorsqu'on les chauffe pour les toute contamination par des ribonu-

cléases. Ceci l'amena, après un deuxième post-doc et son retour en Suède au Karolinska, à s'intéresser à l'instabilité intrinsèque de l'ADN et à montrer que différentes réactions de dépurination, de déamination et de méthylation résultant de la structure chimique de la molécule sont susceptibles, même en l'absence d'agressions extérieures, d'introduire chaque jour des dizaines de milliers de mutations dans un génome humain. Il en conclut à l'existence de mécanismes assurant la réparation de la quasi-totalité de ces anomalies et se consacra dès lors à leur mise en évidence : la découverte en 1974 du système d'excision-réparation [1] devait montrer le bien-fondé de cette approche [2] (Figure 1). Ce système intervient, par exemple, après la déamination de la cytosine, qui transforme cette base en uracile. Le système d'excision-réparation fait intervenir une glycosylase qui détecte et excise l'uracil, puis toute une série de protéines qui éliminent les nucléotides avoisinants (sur le même brin), puis reconstituent la séquence en utilisant le brin opposé comme matrice, et finalement referment l'interruption.

C'était le début de nombreuses études qui révélèrent l'un après l'autre différents systèmes de réparation et décrivirent les protéines impliquées, comme le raconte Lindahl dans My Journey to DNA repair [3]. Après quelques années au Karolinska puis à l'Université de Gothenburg, Lindahl allait rejoindre l'Imperial Cancer Research Fund (ICRF) à Londres, où la connexion entre mécanismes de réparation de I'ADN et biologie des tumeurs fut largement explorée ; il assura à partir de 1986 la direction des laboratoires de l'ICRF à Clare Hall (aujourd'hui Francis Crick Institute), jusqu'en 2005, et prit sa retraite en 2009.

\footnotetext{
${ }^{1}$ Le laboratoire de Fresco s'intéressait aux propriétés des ARNt après dénaturation et renaturation.
} 


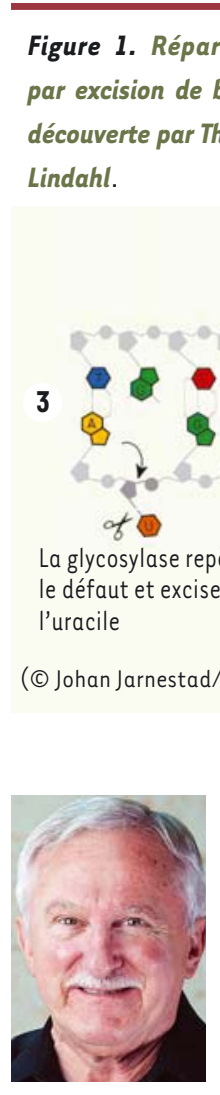

\section{Paul Modrich}

Né en 1946, Paul Modrich est originaire du Nouveau Mexique et fils du professeur de biologie du collège local - lequel fit preuve d'une étonnante clairvoyance en lui recommandant, lorsqu'il avait 17 ans, de s'intéresser à l'ADN'... II suivit cet avis, fréquenta au cours de sa licence à l'Université de Stanford un laboratoire travaillant sur les bactériophages puis obtint en 1973 un doctorat (toujours à Stanford) portant sur les fonctions de l'ADN ligase. À la fin des années 1970 , il s'attaqua au problème de la réparation des erreurs de réplication de l'ADN. On savait que l'ADN polymérase introduit environ une seule erreur tous les 10 millions de bases - belle performance mais qui, lors de la réplication d'un génome humain diploïde, introduirait environ 600 mutations $^{3}$ dans l'ADN nouvellement synthétisé. II devait donc exister un mécanisme de correction de ces anomalies, ce qui supposait que ce système soit capable de reconnaître le brin nouvellement synthétisé afin que la correction se fasse selon la séquence du brin d'origine. Modrich parvint à le mettre en évidence chez Escherichia coli, à le reconstituer dans des extraits acellulaires et à isoler les 11 protéines responsables de cette activité : ce fut la découverte du système MMR (mismatch repair ou « réparation des mésappariements ») [4], dans lequel la reconnaissance du brin d'ADN à rectifier repose sur des méthylations différentielles ou sur l'existence de coupures (nicks) dans le brin nouvellement synthétisé. Ce système améliore la fidélité de la réplication d'un facteur 1000 , aboutissant à moins d'une erreur par génome diploïde lors de la division d'une cellule. Modrich allait montrer un peu plus tard qu'un système analogue existe chez les cellules de mammifères [5] - et que les défauts de ce système peuvent être responsables de l'apparition de cancers, la démonstration la plus frappante de ce fait étant le cancer du côlon héréditaire non polyposique. II continue ses travaux, à Duke University (Durham), sur les protéines de réparation procaryotes et leur emploi pour la manipulation de I'ADN, et, dans le

${ }^{2}$ "You should learn about this DNA stuff», lui dit son père en 1963 , selon le souvenir de Modrich.

${ }^{3} 6 \times 10^{9}$ bases (un génome diploïde) $\times 10^{-7}$ (une erreur toutes les $10^{7}$ bases) soit 600 erreurs de copie. système humain, sur les relations entre réparation et réponse apoptotique dans le cadre des cancers et de leur traitement.

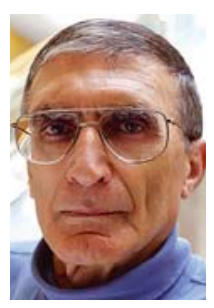

\section{Aziz Sankar}

Le parcours du troisième lauréat, Aziz Sankar (né en 1946 à Savur, Turquie), est de loin le plus original. Ses deux parents étaient analphabètes, et il était le septième d'une fratrie de huit enfants : il parvint néanmoins à faire d'excellentes études, aidé sans doute par le fait que le Parti Démocratique de Menderes, au pouvoir à l'époque, développait considérablement le secteur de l'éducation. Cette remarquable mobilité sociale se poursuivit par des études de médecine à la faculté d'Istanbul où il entra en 1963 (à 17 ans) et dont il sortit major (sur 625) en 1969. II s'y était découvert un vif intérêt pour la recherche en biochimie, mais suivit la recommandation faite par ses enseignants d'exercer quelque temps en milieu rural après l'obtention de son diplôme. Cela fait, il partit en 1971 pour les États-Unis, où il avait été accepté dans le laboratoire de Claud Rupert à l'Université de Texas (Dallas). Ce choix avait été motivé par son intérêt pour le phénomène de photoréactivation, dans lequel l'ADN d'une bactérie endommagé par l'exposition à des radiations ultraviolettes (avec formation de dimères de thymine) peut être réparé par exposition à la lumière bleue. Cette réparation est médiée par une enzyme, la photolyase, découverte quelques années plus tôt par Rupert. Sancar lui consacra sont travail de doctorat, culminant en 1976 avec le clonage du gène performance remarquable en ce tout début de l'ère $d u$ Génie génétique ${ }^{4}$.

Son PhD en poche, Sancar chercha un laboratoire pour poursuivre ses travaux sur la réparation d'ADN dans le cadre d'un post-doc - mais fut refusé par trois laboratoires successifs. Le quatrième, celui de Dean Rupp à l'Université de Yale (New Haven), était bien intéressé par le thème mais ne pouvait lui offrir qu'un emploi de technicien - ce qu'il accepta en 1977. Yale s'avéra être un environnement favorable qui lui permit de cloner plusieurs gènes impliqués dans la réparation d'ADN chez $\varepsilon$. coli et finalement de définir le mécanisme d'excisionréparation des nucléotides ${ }^{5}$ qui permet à la cellule de se débarrasser des dimères de thymine résultant de l'irradiation UV (ultraviolets), lesquels empêcheraient sinon la réplication de l'ADN [6]. Nommé professeur à l'Université

\footnotetext{
${ }^{4}$ «Le premier gène cloné à l'Est des Montagnes Rocheuses », selon Sankar.
}

${ }^{5}$ Différent du système d'excision-réparation de bases découvert par Lindahl. 
de Caroline du Nord (Chapel Hill), Sankar reprit son travail sur la photolyase (dont il parvint finalement à prouver le mécanisme d'action), et montra également l'existence d'un système analogue d'excisionréparation dans les cellules humaines [7]. Comme les gènes impliqués chez l'homme ne présentent aucune homologie avec leurs correspondants bactériens, ceci fournit un joli exemple d'évolution convergente. Plus tard, la découverte un peu fortuite qu'un homologue humain de la photolyase est impliqué dans la régulation du cycle circadien se rattacha au thème précédent grâce à la démonstration que l'horloge circadienne régule l'activité des mécanismes d'excision-réparation - ce qui a des conséquences tant pour la compréhension de l'oncogenèse que pour la définition de régimes de chimiothérapie plus efficaces.

Aziz Sankar, qui est le premier prix Nobel scientifique turc ${ }^{6}$, continue ses travaux à l'Université de Caroline du Nord, notamment sur la réparation de I'ADN dans les cellules cancéreuses en cours de radiothérapie. J'ai décrit son itinéraire avec un peu plus de détails parce qu'il m'a semblé particulièrement remarquable, qu'il montre la marque d'une ténacité et d'un caractère peu communs - et peut-être aussi qu'il témoigne d'une époque où l'accès à l'instruction et la mobilité sociale étaient plus valorisés que de nos jours.

\section{Le bon choix?}

La prise de décision du comité Nobel (qui doit désigner au maximum trois lauréats vivants) a dû être particulièrement difficile : les mécanismes de réparation de l'ADN sont abondants, complexes, et ont fait l'objet des travaux de très nombreux chercheurs. L'éditorial du numéro de novembre de la revue DNA repair [8] se fait l'écho d'une certaine perplexité devant ce choix. Le prestigieux prix Lasker, souvent considéré comme l'antichambre du Nobel, concernait lui aussi cette année la réparation de l'ADN, mais il a récompensé deux autres chercheurs, Evelyn Witkin (Rutgers University, New Brunswick) pour la découverte du système SOS de réparation de l'ADN, et Stephen Elledge (Brigham and Women's Hospital, Boston) pour l'étude de la régulation des systèmes de réparation (DDR, DNA damage response) [9] $(\rightarrow)$. II n'en reste pas moins que les trois mécanismes élucidés par les lauréats du Nobel sont sans doute les plus fondamentaux, et que ces trois chercheurs méritent largement l'honneur qui leur est fait, plusieurs dizaines d'années après leurs découvertes fondamentales. $\diamond$ $(\rightarrow)$ Voir l'article de Judith Miné-Hattab, p. 123 de ce numéro

\section{SUMMARY}

\section{A Nobel Prize for DNA repair}

This year's Nobel Prize for chemistry recognizes the seminal contributions of three researchers who discovered the existence and the basic mechanisms of DNA repair: base excision repair, mismatch repair, and nucleotide excision repair. They have since been joined by many scientists elucidating diverse aspects of these complex mechanisms that now constitute a thriving research field with many applications, notably for understanding oncogenesis and devising more effective therapies.

\section{LIENS D'INTÉRÊT}

L'auteur déclare n'avoir aucun lien d'intérêt concernant les données publiées dans cet article.

\section{RÉFÉRENCES}

1. Lindahl T. New class of enzymes acting on damaged DNA. Nature 1976 ; 259 : 64-6.

2. Lindahl T. Instability and decay of the primary structure of DNA. Nature $1993 ; 362: 709-15$.

3. Lindahl T. My journey to DNA repair. Genomics Proteomics Bioinformatics $2013 ; 11: 2-7$.

4. Su SS, Modrich P. Escherichia coli mutS-encoded protein binds to mismatched DNA base pairs. Proc Natl Acad Sci USA 1986 ; 83 : 5057-61.

5. Modrich P. Mechanisms in eukaryotic mismatch repair. J Biol Chem 2006 ; $281: 30305-9$

6. Sancar A, Rupp WD. A novel repair enzyme: UVRABC excision nuclease of Escherichia coli cuts a DNA strand on both sides of the damaged region. Cell $1983 ; 33: 249-60$.

7. Huang JC, Svoboda DL, Reardon JT, Sancar A. Human nucleotide excision nuclease removes thymine dimers from DNA by incising the 22nd phosphodiester bond 5' and the 6th phosphodiester bond 3' to the photodimer. Proc Natl Acad Sci USA 1992 ; 89 : 3664-8.

8. Wilson SH, Friedberg E. Editorial. DNA Repair (Amst) 2015 ; 35 : v. doi: 10.1016/S1568-7864(15)00215-3.

9. Miné-Hattab J. Prix Lasker 2015 (Evelyn M. Witkin et Stephen J. Elledge). La réparation de l'ADN est à l'honneur. Med Sci (Paris) 2016 ; 32 : 123-4
TIRÉS À PART

B. Jordan

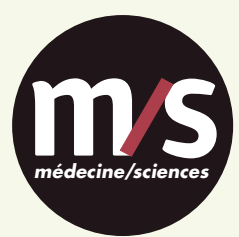

Tarifs d'abonnement $m / s-2016$ Abonnez-vous à médecine/sciences
$>$ Grâce à $\mathrm{m} / \mathrm{s}$, vivez en direct les progrès des sciences biologiques et médicales

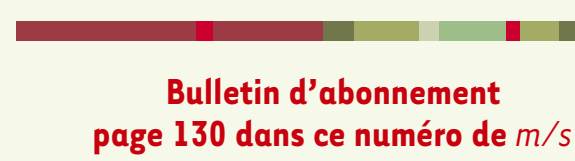

